\title{
Limited evidence available for the impact of school-based behavioural interventions on oral health
}

\author{
Abstracted from \\ Cooper AM, O'Malley LA, Elison SN, et al. \\ Primary school-based behavioural interventions for preventing caries. \\ Cochrane Database Syst Rev 2013; Issue 5. Art. No: CD009378. DOI: 10.1002/14651858.CD009378.pub2. \\ Address for correspondence: Mrs Luisa Fernandez Mauleffinch, Cochrane Oral Health Group, \\ School of Dentistry, The University of Manchester, Coupland III Building, 1st Floor, \\ Oxford Road, Manchester, M13 9PL, UK. E-mail: luisa.fernandez@manchester.ac.uk
}

\section{Question: Can school-based interventions aimed at changing toothbrushing and food consumption habits reduce caries?}

Data sources The Cochrane Oral Health Group's Trials Register, and Cochrane Central Register of Controlled Trials (CENTRAL), Medline, Embase, CINAHL, PsycINFO, Current Controlled Trials, ClinicalTrials. gov, Web of Science and Dissertations and Theses via Proquest databases were searched. A number of relevant journals, (Acta Odontologica Scandinavica, ASDC Journal of Dentistry for Children, British Dental Journal, Caries Research, Community Dental Health, Community Dentistry and Oral Epidemiology, Journal of the American Dental Association, Journal of Dental Research, Journal of Public Health Dentistry, Swedish Dental Journal, International Journal of Paediatric Dentistry) not already searched as part of the Cochrane Journal Handsearching Programme were handsearched. There were no restrictions regarding language or date of publication.

Study selection Randomised controlled trials (RCTs) where randomisation occured at the level of the group (cluster by school and/ or class) or individual children were included. Included studies had to include behavioural interventions addressing both toothbrushing and consumption of cariogenic foods or drinks and have a primary school as a focus for delivery of the intervention.

Data extraction and synthesis Two pairs of review authors independently extracted data related to methods, participants, intervention design including behaviour change techniques (BCTs) utilised, outcome measures and risk of bias. A qualitative synthesis was conducted.

Results Four studies involving a total of 2302 children were included. One study was at unclear risk of bias and three were at high risk of bias. The studies were heterogeneous in both intervention and outcome measures and also suffered from poor reporting. Only one included study reported caries development as an outcome. This small study at unclear risk of bias showed a prevented fraction of 0.65 (95\% confidence interval $(\mathrm{Cl}) 0.12$ to 1.18 ) in the intervention group. However, as this is based on a single study, this finding should

This paper is based on a Cochrane Review published in the Cochrane Library 2013, issue 5 (see www.thecochranelibrary.com for information). Cochrane Reviews are regularly updated as new evidence emerges and in response to feedback, and the Cochrane Library should be consulted for the most recent version of the review. be interpreted with caution. All three studies that reported plaque outcomes found statistically significant reduction in plaque in the intervention groups, but due to differences in plaque reporting between studies these could not be combined. Two of these studies included an active home component where parents were given tasks relating to the school oral health programme, (games and homework) to complete with their children. Secondary outcome measures from one study reported that the intervention had a positive impact upon children's oral health knowledge.

Conclusions Currently, there is insufficient evidence for the efficacy of primary school-based behavioural interventions for reducing caries. There is limited evidence for the effectiveness of these interventions on plaque outcomes and on children's oral health knowledge acquisition. None of the included interventions were reported as being based on or derived from behavioural theory. There is a need for further high quality research to utilise theory in the design and evaluation of interventions for changing oral health related behaviours in children and their parents.

\section{Commentary}

This Cochrane review looked at the effect on caries prevention of school-based interventions aimed at changing behaviour related to toothbrushing habits and the frequency of consumption of cariogenic food and drink in children, specifically in the four to 12 -year-old range. The evidence that toothbrushing with fluoride toothpaste reduces caries is unequivocal ${ }^{1}$ and although brushing at least twice a day is optimum, many children do not achieve this. Consequently, school-based programmes offer not only an opportunity to undertake an effective procedure but also an opportunity to learn a key life skill that could be translated into behaviour at home.

For inclusion in this review studies were required to have a focus around toothbrushing and cariogenic foods, to use schools as the focal site for intervention delivery and to contain skills, instructions and educational components. The interventions could be delivered by a range of staff. Only four studies met the criteria as the majority of studies initially identified were either not randomised or did not include any food based intervention.

The review methodology followed the usual robust Cochrane methodology and the main outcome measure was caries. The minimally accepted length of follow-up for trials where the outcome is caries increment is 12 months and only two of the included studies were longer than this, only one of which reported on dmfs/DMFS. 
Three studies reported plaque scores but substantial heterogeneity was reported by the authors so the analysis was not presented. Only one of the studies reported non-clinical outcomes, which were self reported.

The evidence available for this review is limited in its ability to provide answers to the question of how to prevent caries using school-based interventions and there was a lack of detail provided about the interventions in the studies themselves.

As the authors note, there has been increased interest and improvement in behaviour change techniques in the past decade, ${ }^{2}$ and a recent review by Harris et al. ${ }^{3}$ found some evidence that one-to-one dietary interventions in the dental setting can change behaviour, although the evidence is greater for interventions aiming to change fruit/vegetable and alcohol consumption than for those aiming to change dietary sugar consumption. In the Harris review the main outcome under consideration were dietary behaviour changes rather than caries as in this current review. Mitchie in her paper argues that what is required is clarity about the 'behaviour' being the defined and measured outcome of the interventions rather than other outcomes up (or down) the change.

\section{Derek Richards} Centre for Evidence-based Dentistry, Oxford, UK.

1. Marinho VCC, Higgins JPT, Sheiham A, Logan S. Fluoride toothpastes for preventing dental caries in children and adolescents. Cochrane Database Syst Rev 2003; Issue 1. Art. No. CD002278. DOI: 10.1002/14651858.CD002278.

2. Michie S, Johnston $M$. Theories and techniques of behaviour change: Developing a cumulative science of behaviour change. Health Psychol Rev 2012; 6: 1-6.

3. Harris R, Gamboa A, Dailey Y, Ashcroft A. One-to-one dietary interventions undertaken in a dental setting to change dietary behaviour. Cochrane Database Syst Rev. 2012; Issue 3. Art. No. CD006540. DOI: 10.1002/14651858.CD006540.pub2.

Evidence-Based Dentistry (2013) 14, 42-43. doi:10.1038/sj.ebd.6400929 